Editorial

\section{Metabolism Tailors Macrophage Functions: One Size Does Not Fit All}

\author{
Alessandra Castegna ${ }^{1,2, *}$, Massimiliano Mazzone ${ }^{3,4, *}$ \\ 1 Department of Biosciences, Biotechnologies and Biopharmaceutics, University \\ of Bari, Bari 70125, Italy \\ 2 IBIOM-CNR, Institute of Biomembranes, Bioenergetics and Molecular \\ Biotechnologies, National Research Council, Bari 70125, Italy \\ 3 Laboratory of Tumor Inflammation and Angiogenesis, Center for Cancer \\ Biology, Department of Oncology, KU Leuven, Leuven B3000, Belgium \\ 4 Laboratory of Tumor Inflammation and Angiogenesis, Center for Cancer \\ Biology, VIB, Leuven B3000, Belgium \\ * Correspondence: Alessandra Castegna, Email: alessandra.castegna@uniba.it; \\ Massimiliano Mazzone, Email: massimiliano.mazzone@kuleuven.vib.be.
}

It is well established that macrophages are critical for maintaining tissue integrity. It follows that impaired or exacerbated macrophage functions are often associated to disease. This is true in inflammatory related disorders, such as obesity, in which tissue macrophages become dysfunctional and display a persistent inflammatory activity. Conversely, in cancer, macrophages acquire an anti-inflammatory, immunosuppressive and pro-angiogenic function, sustaining, rather than constraining, tumor development and metastasis formation [1,2]. In all these pathological conditions macrophages receive signals from the surrounding tissues, engaging in a very complex plethora of functional states that support disease. Emerging research is now showing that in vitro polarized macrophages display different metabolic features, which are associated to their effector functions [3,4]. Yet, it is not completely clear if this holds true in vivo, and if specific metabolic traits impose a defined phenotype or vice versa, since the in vivo complexity of macrophage heterogeneity together with the impact that environmental signals can have on their phenotypic skewing would require a temporal and spatial definition that is strongly awaited. The present collection aims at providing an effective tool to contribute to the comprehensive understanding of the immunometabolic functions of macrophages and their communication with tissues in vivo in the context of two specific diseases: obesity and cancer.

Obesity is accompanied by a chronic inflammatory process including the recruitment of monocyte and differentiation in inflammatory adipose tissue macrophages (ATMs). ATMs contribute to an inflammatory state of the adipose tissue but display heterogeneous phenotypes, reflecting profound changes in their local microenvironment, including adipocyte apoptosis and spill-over of their lipid content [2]. Targeting ATM phenotypes to ameliorate inflammation in obesity might represent a 
strategy to fight the systemic consequences of this condition. To do so it is important to dissect the specific signalling and metabolic cues responsible for the acquisition of undesired functions. Different (sometimes divergent) microenvironmental signals are responsible for the acquisition of specific tissue-derived macrophage subpopulations. Korf and coworkers [5] provide an extensive review on this topic, by mapping the different ATM subsets, and their associated metabolic and functional profiles, together with some speculation on their potential contribution to homeostatic or pathological conditions associated to obesity.

The research article of Blaszczak and coworkers [6] introduces the concept of obesogenic memory, indicating that persistent inflammation may occur independently of body weight normalization. The authors address the relationship between previous obesity and adipose tissue inflammation, in which immunosuppressive regulatory $\mathrm{T}$ cells (Tregs), phagocytic and anti-inflammatory (M2-like) macrophages, and innate lymphoid cells type 2 (ILC2) are lost leading to insulin resistance (IR). After 6 months of high fat diet (HFD) in male C57/Bl6 mice, a normal chow diet fails in normalizing IR and glucose intolerance despite body weight and total fat reduction. At a cellular level, these unresolved systemic issues are mirrored by persistent abnormalities in the content of adipocytes, ATassociated Tregs, $\mathrm{CD} 8^{+} \mathrm{T}$ cells, ILC2 cells, and pro-inflammatory (M1-like) macrophages, leading to persistent AT inflammation.

In the effort of identifying specific targets to tackle the aberrant macrophage switch occurring in obesity, Sharma and coworkers [7] provide experimental evidence for the role of Netrin-1 in mediating inflammation by altering the phenotypic fate of ATMs. Using a conditional, macrophage specific gene deletion approach, they elegantly demonstrate that targeting Netrin-1 in macrophages reprograms the ATM phenotype in obesity, leading to reduced adipose inflammation, and improved lipid handling and metabolic function. This important result opens the possibility for the pharmacological exploitation of this target to improve the inflammatory component of the complex disease associated to obesity.

Shifting to cancer, Santos and coworkers [8] give an overview on the microenvironmental metabolic signals underlining the acquisition of an immunosuppressive phenotype of tumor-associated macrophages (TAMs), identifying in lactate, initially considered a simple by-product of malignant highly glycolytic cancer cells, a major cross-talk mediator of macrophage functions. This review focuses on key molecular mechanisms by which lactate metabolically modulates immune cell response during tumor development and progression.

As macrophage functions strongly associate to specific metabolic signatures, mitochondrial metabolism is possibly a strong determinant of the macrophage functional state as it orchestrates whole cell metabolic responses. Recent emerging evidence is showing that the mitochondrial respiratory chain is a flexible bioenergetic source that can be controlled by innate immunity [9]. In the context of an inflammatory response to a 
pathogen, it is debated how the different complexes plastically respond and assemble into supercomplex structures to satisfy the stress and fuel demand associated to inflammation. Escoll and coworkers [10] review the different theories and body of evidence describing the regulation of ETC complexes and super-complexes during infection, with the aim of providing clues on the mechanisms by which the mitochondrial OXPHOS machinery is rearranging in order to meet the rapid metabolic demand associated to a pathogen infection.

In conclusion, by understanding the metabolic heterogeneity of pathways underlining a specific inflammatory reaction, it may become possible to control such a response and reach tailored therapies that are more and more specific for a pathological condition without harnessing the homeostasis of other tissues or without dampening important immunological barriers.

\section{CONFLICTS OF INTERESTS}

The authors do not have conflicts of interest to disclose.

\section{REFERENCES}

1. Flerin NC, Pinioti S, Menga A, Castegna A, Mazzone M. Impact of Immunometabolism on Cancer Metastasis: A Focus on $\mathrm{T}$ Cells and Macrophages. Cold Spring Harb Perspect Med. 2020;10(9):a037044.

2. Castegna A, Gissi R, Menga A, Montopoli M, Favia M, Viola A, et al. Pharmacological targets of metabolism in disease: Opportunities from macrophages. Pharmacol Ther. 2020;210:107521.

3. Viola A, Munari F, Sánchez-Rodríguez R, Scolaro T, Castegna A. The Metabolic Signature of Macrophage Responses. Front Immunol. 2019;10:1462.

4. Mazzone M, Menga A, Castegna A. Metabolism and TAM functions-it takes two to tango. FEBS J. 2018;285(4):700-16.

5. Korf H, Boesch M, Feio-Azevedo R, Smets L, Vandecasteele R, van der Merwe S. Depicting the Landscape of Adipose Tissue-Specific Macrophages and Their Immunometabolic Signatures during Obesity. Immunometabolism. 2020;2(1):e200001. https://doi.org/10.20900/immunometab20200001

6. Blaszczak AM, Bernier M, Wright VP, Gebhardt G, Anandani K, Liu J, et al. Obesogenic Memory Maintains Adipose Tissue Inflammation and Insulin Resistance. Immunometabolism. 2020;2(3):e200023. https://doi.org/10.20900/immunometab20200023

7. Sharma S, Schlegel PM, Brown EJ, Sansbury BE, Weinstock A, Afonso M, et al. Netrin-1 Alters Adipose Tissue Macrophage Fate and Function in Obesity. Immunometabolism. 2019;1:e190010. https://doi.org/10.20900/immunometab20190010

8. Santos N, Pereira-Nunes A, Baltazar F, Granja S. Lactate as a Regulator of Cancer Inflammation and Immunity. Immunometabolism. 2019;1:e190015. https://doi.org/10.20900/immunometab20190015

9. Benmoussa K, Garaude J, Acín-Pérez R. How Mitochondrial Metabolism Contributes to Macrophage Phenotype and Functions. J Mol Biol. 
2018;430(21):3906-3921.

10. Escoll P, Platon L, Buchrieser C. Roles of Mitochondrial Respiratory Complexes during Infection. Immunometabolism. 2019;1:e190011. https://doi.org/10.20900/immunometab20190011

How to cite this article:

Castegna A, Mazzone M. Metabolism Tailors Macrophage Functions: One Size Does Not Fit All. Immunometabolism. 2020;2(4):e200033. https://doi.org/10.20900/immunometab20200033 